\title{
ALMOST EVERYWHERE DIVERGENCE OF MULTIPLE WALSH-FOURIER SERIES
}

\author{
DAVID C. HARRIS
}

(Communicated by Richard R. Goldberg)

\begin{abstract}
C. Fefferman $[1,2,3]$ has shown that the multiple Fourier series of an $f \in L^{p}, p<2$, may diverge a.e. when summed over expanding spheres, but converges a.e. when summed over expanding polyhedral surfaces. We show this dichotomy does not prevail for multiple Walsh-Fourier series.
\end{abstract}

1. Introduction. Let $\psi_{0}, \psi_{1}, \ldots$ denote the Walsh-Paley system, $G$ denote the dyadic group (see Fine [4]) and set

$$
\psi_{m, n}(x, y)=\psi_{m}(x) \psi_{n}(y) \quad \text { for } x, y \in G ; m, n \in \hat{G} .
$$

For any function $f$ integrable on $G \times G$ let

$$
\hat{f}(m, n)=\int_{G} \int_{G} f \psi_{m, n}
$$

represent the double Walsh-Fourier coefficients of $f$. Given a region $S$ in $[0, \infty) \times$ $[0, \infty)$ with piecewise $C^{1}$ boundary and a function $f$ integrable on $G \times G$, let

$$
S_{\gamma} f=\sum_{(m, n) \in \gamma S \cap \hat{G} \times \hat{G}} \hat{f}(m, n) \psi_{m, n}
$$

where $\gamma>0$ and $\gamma S$ represents dilation of $S$ by $\gamma$. If $S$ represents a quarter disc in the first quadrant then $S_{\gamma} f$ is a circular sum of the double Walsh-Fourier series of $f$. If $S$ represents a region with polygonal boundary then $S_{\gamma}$ is a polygonal sum of the double Walsh-Fourier series of $f$. In $\S 3$ we shall prove the following result.

THEOREM 1 . If $S$ is a region $[0, \infty) \times[0, \infty)$ with piecewise $C^{1}$ boundary not always parallel to the axes, and if $p<2$, then there exists an $f \in L^{p}(G \times G)$ such that $S_{\gamma} f$ diverges a.e. and in $L^{p}$ norm as $\gamma \rightarrow \infty$.

In particular, for double Walsh-Fourier series of functions in $L^{p}, p<2$, neither circular sums, nor regular polygonal sums (other than square sums) need converge a.e. The case of square partial sums is still unresolved. We only know that square partial sums converge a.e. when $f \in L^{2}(G \times G)$. We will also extend Theorem 1 to $N$-dimensions.

Received by the editors July 14, 1986.

1980 Mathematics Subject Classification (1985 Revision). Primary 42C10; Secondary 43A50, 43A75.

Key words and phrases. Walsh functions, multiple Fourier series, a.e. divergence, multipliers.

This research was completed for partial satisfaction of requirements for a Ph.D. degree at the University of Tennessee, Knoxville.

This research was partially supported by the National Science Foundation under grant INT8400708. 
2. A multiplier problem. Our proof of Theorem 1 uses the following solution to a multiplier problem.

THEOREM 2. Let $\sigma>0, \beta$ be any real, $f \in L^{1}(G \times G)$, and

$$
T_{\sigma, \beta} f=\sum_{n<\sigma m+\beta} \hat{f}(m, n) \psi_{m, n} .
$$

Then $T_{\sigma, \beta}$ is unbounded as an operator on $L^{p}(G \times G)$ for all $1 \leq p \leq \infty, p \neq 2$.

This theorem was known for the special case $\sigma=1, \beta=0$ (see Wo-Sang Young [7]). She used it to show that the trigonometric and Walsh system are not equivalent bases in $L^{p}, p \neq 2$. Our proof uses different techniques.

For the case $\sigma \neq 1, \beta=0$ it is necessary to construct certain subgroups of $\hat{G} \times \hat{G}$ which lie near the line $y=\sigma x$. This construction is accomplished in Lemma 1 below. First we introduce some notation and discuss preliminaries. Let $M$ be a positive integer. Let increasing powers of 2 be given:

$$
A_{0}=2^{l_{0}}, A_{1}=2^{l_{1}}, \ldots, A_{M-1}=2^{l_{M-1}} \quad \text { with } 0 \leq l_{0}<\cdots<l_{M-1} .
$$

Let positive integers $s_{j}$ be given for $j=0, \ldots, M-1$. Suppose that

$$
s_{j} A_{j}<A_{j+1} \text { for } j=0, \ldots, M-2 .
$$

For any nonnegative integer $a<2^{M}$ and $j=0, \ldots, M-1$ let $a_{j}=0$ or 1 be determined by the binary expansion of $a, a=\sum_{j=0}^{\infty} a_{j} 2^{j}$. Define $\Gamma_{0}=\Gamma_{0}\left(M, A_{j}, s_{j}\right)$ to be $\left\{\sum_{i=0}^{M-1} a_{j} s_{j} A_{j} \mid a=0,1, \ldots, 2^{M}-1\right\}$. One may easily verify $\Gamma_{0}$ is a subgroup of $\hat{G}$. Indeed (1) implies for $a, b<2^{M}$ that

$$
\left(\sum_{j=0}^{M-1} a_{j} s_{j} A_{j}\right)+\left(\sum_{j=0}^{M-1} b_{j} s_{j} A_{j}\right)=\sum_{j=0}^{M-1}\left(a_{j} \dot{+} b_{j}\right) s_{j} A_{j} .
$$

(For $\dot{+}$ see e.g. Fine [1].) Therefore, in fact, $a \rightarrow \sum_{j=0}^{M-1} a_{j} s_{j} A_{j}$ is a $\dot{+}$-isomorphism from $\left\{0, \ldots, 2^{M}-1\right\}$ onto $\Gamma_{0}$. Suppose in addition to (1) we are given positive integers $t_{j}$ for $j=0, \ldots, M_{1}$ such that $t_{j} A_{j}<A_{j+1}$ for $j=0, \ldots, M-2$. It is then immediate that

$$
\Gamma=\Gamma\left(M, A_{j}, s_{j}, t_{j}\right)=\left\{\sum_{j=0}^{M-1} a_{j}\left(s_{j}, t_{j}\right) A_{j} \mid a=0, \ldots, 2^{M}-1\right\}
$$

is a subgroup of $\hat{G} \times \hat{G}$ where $a_{j}\left(s_{j}, t_{j}\right) A_{j}=\left(a_{j} s_{j} A_{j}, a_{j} t_{j} A_{j}\right)$. Furthermore $a \rightarrow$ $\sum_{j=0}^{M-1} a_{j}\left(s_{j}, t_{j}\right) A_{j}$ is a $\dot{+}$-isomorphism from $\left\{0, \ldots, 2^{M}-1\right\}$ onto $\Gamma$.

LEMMA 1. Let $M$ be a positive integer, $A_{0}$ be any power of 2 , and let $\sigma>0$ and $\varepsilon>0$ be given. Then there is a subgroup $\Gamma=\Gamma\left(M, A_{j}, s_{j}, t_{j}\right) \subset \hat{G} \times \hat{G}$ such that

$$
|n-\sigma m|<\varepsilon \quad \text { for all }(m, n) \in \Gamma \text {. }
$$


To prove Lemma 1, we use a classical theorem of rational approximation:

THEOREM (KRONECKER) (SEE ZYGMUND [8]). Given any real number $\sigma$ there are infinitely many fractions $t / s$ such that $|\sigma-t / s| \leq s^{-2}$.

ProOf of LemMa 1. Use induction on $M$. Assume first that $M=1$. Let $A_{0}$ be an arbitrary power of 2 . By Kronecker's theorem there are integers $s_{0}, t_{0}$ such that $s_{0}>A_{0} / \varepsilon$ and such that $\left|\sigma-t_{0} / s_{0}\right| \leq s_{0}^{-2}$. It follows that $\left|t_{0}-\sigma s_{0}\right|<\varepsilon / A_{0}$ and thus $\left|A_{0} t_{0}-\sigma s_{0} A_{0}\right|<\varepsilon$. Thus we may let $\Gamma=\Gamma\left(1, A_{0}, s_{0}, t_{0}\right)$ to finish the case $M=1$. Now assume $M>1$. Let $A_{0}$ be given. Assume by induction that $\Gamma^{\prime}=\Gamma^{\prime}\left(M-1, A_{j}, s_{j}, t_{j}\right)$ is given satisfying

$$
|n-\sigma m|<\varepsilon / 2 \text { for all }(m, n) \in \Gamma^{\prime} .
$$

Choose $A_{M-1}$, a power of 2 , so large that $s_{M-2} A_{M-2}<A_{M-1}$ and $t_{M-2} A_{M-2}<$ $A_{M-1}$. Applying Kronecker's theorem again, we may choose integers $s_{M-1}, t_{M-1}$ such that

$$
\left|t_{M-1} A_{M-1}-\sigma s_{M-1} A_{M-1}\right|<\varepsilon / 2 .
$$

Set $\Gamma=\Gamma\left(M, A_{j}, s_{j}, t_{j}\right)$ and note that $\Gamma=\Gamma^{\prime} \cup\left(\Gamma^{\prime} \dot{+}\left(s_{M-1} A_{M-1}, t_{M-1} A_{M-1}\right)\right)$. Furthermore since $A_{M-1}$ is a power of 2 and $A_{M-1}>m, n$ for any $(m, n) \in \Gamma^{\prime}$ we have $(m, n) \dot{+}\left(s_{M-1} A_{M-1}, t_{M-1} A_{M-1}\right)=(m, n)+\left(s_{M-1} A_{M-1}, t_{M-1} A_{M-1}\right)$. It follows that $|n-\sigma m|<\varepsilon$ for any $(m, n) \in \Gamma$. By induction the proof of Lemma 1 is complete.

REMARK. If $\sigma=t / s$ is rational, then we may take $s_{j}=s, t_{j}=t$, and $A_{j}=A^{j}$ where $A$ is some fixed power of 2 greater than $s$ and $t$. In this case, $\Gamma$ lies exactly on the line $y=\sigma x$ and Kronecker's theorem is not needed.

Given a subgroup $\Gamma$ of $\hat{G} \times \hat{G}$, the annihilator $H$ of $\Gamma$ is defined by

$$
H=\operatorname{ann}(\Gamma)=\{(x, y) \in G \times G: \psi(x, y)=1 \text { for all } \psi \in \Gamma\}
$$

(see Rudin [5]). It is well known that $H$ is a closed subgroup of $G \times G$ and that

and

$$
|H|=\operatorname{meas} H=\frac{1}{\operatorname{card}(\Gamma)}
$$

$$
\begin{aligned}
& \operatorname{ann}(\Gamma) \cap \operatorname{ann}\left(\Gamma^{\prime \prime}\right)=\operatorname{ann}\left(\left\langle\Gamma, \Gamma^{\prime}\right\rangle\right), \text { where }\left\langle\Gamma, \Gamma^{\prime}\right\rangle \\
& \text { denotes the group generated by } \Gamma \cup \Gamma^{\prime} .
\end{aligned}
$$

In the one-dimensional case when $\Gamma=\left\{0,1, \ldots, 2^{M}-1\right\}$, the annihilator of $\Gamma$ is $H=\left[0,2^{-M}\right)$ and the sum of characters in $\Gamma$ is the Dirichlet kernel $D_{2^{M}}$ which satisfies $D_{2 M}=\chi_{H} /|H|$ where $\chi_{H}$ denotes the characteristic function of $H$. A similar calculation subsists for any finite subgroup $\Gamma$ of $\hat{G} \times \hat{G}$ :

LEMMA 2. Let $\Gamma$ be a finite subgroup in $\hat{G} \times \hat{G}$. Then $\sum_{\psi \in \Gamma} \psi=\chi_{H} /|H|$ where $H=\operatorname{ann}(\Gamma)$.

ProOF. That $\sum_{\psi \in \Gamma} \psi=1 /|H|$ for $x \in H$ follows from (2) and the definition of $H$. By orthogonality and by (2) we obtain that $\left\|\sum_{\psi \in \Gamma} \psi\right\|_{2}=\operatorname{card}(\Gamma)=1 /|H|$. But also

$$
\left\|\sum_{\psi \in \Gamma} \psi\right\|_{2}^{2}=\int_{H} \frac{1}{|H|^{2}}+\int_{G \times G \backslash H}\left|\sum_{\psi \in \Gamma} \psi\right|^{2}=\frac{1}{|H|}+\int_{G \times G \backslash H}\left|\sum_{\psi \in \Gamma} \psi\right|^{2}
$$


It follows that $\int_{G \times G \backslash H}\left|\sum_{\psi \in \Gamma} \psi\right|^{2}=0$. Thus $\sum_{\psi \in \Gamma} \psi=0$ for $x \notin H$. The proof is finished.

In regard to $\Gamma$ as in Lemma 1 and its annihilator $H$ we need the following lemma.

LEMMA 3. Let $\Gamma=\Gamma\left(M, A_{j}, s_{j}, t_{j}\right)$ be given, $H$ be the annihilator of $\Gamma$, and $R_{j}(x, y)=\psi_{0, t_{j} A_{j}}(x, y)=\psi_{t_{j} A_{j}}(y)$ or $j=0, \ldots, M-1$ and $(x, y) \in G \times G$. Then $\left\{R_{j}\right\}$ remain orthogonal when restricted to $H$.

ProOF. Let $j, k=0, \ldots, M-1$ and $j \neq k$. Then we have by Lemma 2

$$
\int_{H} R_{j} R_{k}=\int_{G \times G} \chi_{h} R_{j} R_{k}=|H| \sum_{\psi \in \Gamma} \int_{G \times G} \psi \psi_{0, t_{j} A_{j}+t_{k} A_{k}} .
$$

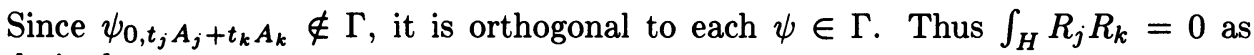
desired.

To prove Theorem 2 it is enough by $L^{p}$-duality to consider the case $p<2$. Set $\varepsilon=1 / 2$. For any positive integer $M$, choose $\Gamma=\Gamma\left(M, A_{j}, s_{j}, t_{j}\right)$ according to Lemma 1. For $a=0, \ldots, 2^{M}-1$ let $J(a)$ denote $\sum_{j=0}^{M-1} a_{j}\left(s_{j}, t_{j}\right) A_{j}$. Let $\Gamma_{j}=\left\{J(a) \in \Gamma \mid a_{j}=0\right\}$ for $j=0, \ldots, M-1$. Let $H=\operatorname{ann}(\Gamma)$ and $H_{j}=\operatorname{ann}\left(\Gamma_{j}\right)$. Let $f_{j}=f_{j}^{M}=R_{j} \sum_{\psi \in \Gamma} \psi$. By Lemma 2 we have

$$
f_{j}=R_{j} \frac{\chi_{H}}{|H|} .
$$

Let $f=f^{M}=\sum_{j=0}^{M-1} f_{j}^{M}$. By (4) and Lemma 3 we obtain that

$$
\begin{aligned}
\|f\|_{p} & =\left(\int_{H}\left|\sum_{j=0}^{M-1} f_{j}\right|^{p}\right)^{1 / p}=\frac{1}{|H|}\left(\int_{H}\left|\sum_{j=0}^{M-1} R_{j}\right|^{p}\right)^{1 / p} \\
& \leq \frac{1}{|H|} \cdot|H|^{1 / p-1 / 2}\left(\int_{H}\left|\sum_{j=0}^{M-1} R_{j}\right|^{2}=|H|^{1 / 2} \cdot M^{1 / 2}\right.
\end{aligned}
$$

where $1 / q=1-1 / p$. We now let $T=T_{\sigma, 0}$ and compute $T f$. First note from the definition of $R_{j}$ and $f_{j}$ that

$$
f_{j}=\sum_{a=0}^{2^{M}-1} \psi_{J(a) \dot{+}\left(0, t_{j} A_{j}\right)}
$$

Also note that

$$
\begin{aligned}
J(a) \dot{+}\left(0, t_{j} A_{j}\right) & =J(a)+\left(0, t_{j} A_{j}\right) & & \text { if } J(a) \in \Gamma_{j}, \\
& =J(a)-\left(0, t_{j} A_{j}\right) & & \text { if } J(a) \notin \Gamma_{j} .
\end{aligned}
$$

Since $|n-\sigma m|<\frac{1}{2}$ for $J(a)=(m, n)$ and $a=0, \ldots, 2^{M}-1$; we see that $J(a)+\left(0, t_{j} A_{j}\right)$ is above the line $y=\sigma x$ if $J(a) \in \Gamma_{j}$ and is below the line $y=\sigma x$ if $J(a) \notin \Gamma_{j}$. Thus

$$
T f_{j}=R_{j} \sum_{\psi \in \Gamma \sim \Gamma_{j}} \psi \text { and }\left|T f_{j}\right|=\left|\sum_{\psi \in \Gamma \sim \Gamma_{j}} \psi\right|=\frac{1}{\left|H_{j}\right|} \chi_{H_{j}}
$$


by Lemma 2. Clearly $\left\langle\Gamma_{j}, \Gamma_{k}\right\rangle$ if $j \neq k$, so by (3) $H_{j} \cap H_{k}=H$. By (2) $\left|H_{j}\right|=2|H|$ and thus $\left|H_{j} \sim H\right|=|H|$ for $j=0,1, \ldots, m-1$. Hence

$$
|T f|=\left|\sum_{j=0}^{M-1} T f_{j}\right|=\left\{\begin{array}{l}
? \text { on } H \text { (not needed below), } \\
\frac{1}{\left|H_{j}\right|}=\frac{1}{2|H|} \quad \text { on } H_{j} \sim H, \\
0 \text { otherwise. }
\end{array}\right.
$$

Thus

$$
\left(\int_{G \times G}|T f|^{p}\right)^{1 / p} \geq\left(\sum_{j=0}^{M-1}\left(\frac{1}{2|H|}\right)^{p}|H|\right)^{1 / p}=\frac{1}{2}|H|^{-1 / q} M^{1 / p} .
$$

So $\left\|T f^{M}\right\|_{p} /\left\|f^{M}\right\|_{p} \geq \frac{1}{2} M^{1 / p-1 / 2}$. Since $p<2$, this last quantity approaches $\infty$ as $M \rightarrow \infty$. The unboundedness of $T$ on $L^{p}(G \times G)$ follows for $p \neq 2$. Theorem 2 is proved in the case $\beta=0$.

For the case $\beta \neq 0$ we use a coset of an appropriate $\Gamma$ rather than $\Gamma$ itself. Let $A_{0}$ be a power of 2 with $A_{0}>\max (2, \beta)$. Let a positive integer $M$ be given. According to Lemma 1 choose $\Gamma\left(M, A_{j}, S_{j}, t_{j}\right)$ close to the line $y=\sigma x$, say the $y$-coordinates of $\Gamma\left(M, A_{j}, s_{j}, t_{j}\right)$ differ from the line $y=\sigma x$ by less than 1 . Assume first that $\beta>0$. Let $k=$ greatest integer in $\beta$. Since $A_{0}>k$ it follows that $(0, k) \dot{+} \Gamma=(0, k)+\Gamma$ and that $(0, k)+\Gamma$ lies close to the line $y=\sigma x+\beta$; at least, the $y$-coordinates of $(0, k)+\Gamma$ differ from those of $y=\sigma x+\beta$ by less than 2. By examining (5) and noting that $t_{j} A_{j}>2$ we easily see that $\left(0, t_{j} A_{j}\right) \dot{+}(0, k) \dot{+} \Gamma_{j}$ lies above $y=\sigma x+\beta$ while $\left(0, t_{j} A_{j}\right)+(0, k) \dot{+}\left(\Gamma \sim \Gamma_{j}\right)$ lies below $y=\sigma x+\beta$. Thus

$$
\psi_{0, k} T_{\sigma}\left(R_{j} \sum_{\psi \in \Gamma} \psi\right)=T_{\sigma, \beta}\left(\psi_{0, k} R_{j} \sum_{\psi \in \Gamma} \psi\right)
$$

Hence the case $\beta>0$ is seen to follow from the case $\beta=0$. The case $\beta<0$ can be treated similarly by interchanging $x$ and $y$. The proof of Theorem 2 is complete.

3. A proof of Theorem 1. Let $S$ be a region in the first quadrant with boundary not always parallel to the axes. For $p \leq 2$ the maximal function

$$
S^{*} f=\sum_{\gamma>0}\left|S_{\gamma} f\right| \quad\left(f \in L^{p}(G \times G)\right)
$$

completely determines whether $S_{\gamma} f$ converges a.e. or not. Indeed, Stein [6] has shown that $S_{\gamma} f$ converges a.e. for all $f \in L^{p}(G \times G)$ if and only if $S^{*}$ is of weak type $(p, p)$. In particular, by known interpolation arguments (see e.g. [8]) the proof of Theorem 1 will be complete if we construct polynomials $f_{0}=f_{0}^{M}$ and $\gamma=\gamma_{M}$ for large $M$ which satisfy

$$
\left\|S_{\gamma} f_{0}\right\|_{p}>\frac{1}{2} M^{1 / p-1 / 2}\|f\|_{p} \quad \text { for all } p<2 .
$$

By hypothesis there is some point $(x, y)$ on $\partial S$ such that the tangent line at $(x, y)$ has slope $\tau= \pm \sigma$ with $\sigma>0$. Choose $f$ according to Theorem 2 so that

$$
\left\|T_{\sigma, 0} f\right\|_{p} \geq \frac{1}{2} M^{1 / p-1 / 2}\|f\|_{p} \quad \text { for all } p<2
$$

and so that $\hat{f}(m, n)=0$ when $m$ or $n \geq 2^{N_{M}}$ for some positive integer $N_{M}$. As $\gamma$ becomes large $\partial(\gamma S)$ will become near its tangent line at $(\gamma x, \gamma y)$ of slope $\tau$ over 
an interval of length $2^{N_{M}+1}$. In the case $\tau=\sigma$ one may thus choose appropriate

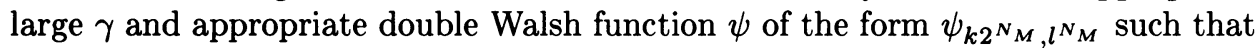
$S_{\gamma}(\psi f)=\psi T_{\sigma, 0}(f)$. Thus in this case $f_{0}=\psi f$ satisfies (6). If $\tau=-\sigma$, it is convenient to vary the point $(x, y)$ if necessary to assume that $y / x$ is rational. We also use the fact that

$$
\left(\psi_{2^{N_{M}}-1,0} f\right)^{-}(m, n)=\hat{f}\left(2^{N_{M}}-1-m, n\right)
$$

for $m<2^{N_{M}}$. We then may obtain that

$$
S_{\gamma}\left(\psi \psi_{2^{N_{M}-1,0}} f\right)=\psi \psi_{2^{N_{M}-1,0}} T_{\sigma, 0} f
$$

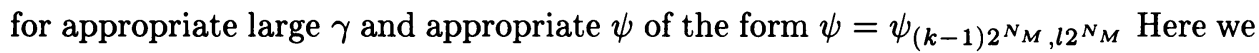
choose $k$ and $l$ with $l / k=y / x$ so that the tangent line at $(\gamma x, \gamma y)$ passes through $\left(k 2^{N_{M}}, l 2^{N_{M}}\right)$ for appropriate $\gamma$. Thus in this case $f_{0}=\psi \psi_{2^{N_{M}-1,0}} f$ satisfies (6). Theorem 1 is proved.

The following $N$-dimensional version of Theorem 1 can be deduced from Theorem 2.

THEOREM $1^{\prime}$. If $S$ is a region in the $N$-fold product $[0, \infty) \times \cdots \times[0, \infty)$ with piecewise $C^{1}$ boundary not always parallel to the coordinate axes, and if $p<2$, then there exists an $f \in L^{p}\left(G^{N}\right)$ such that the $N$-dimensional $S_{\gamma} f$ diverges a.e. and in $L^{p}$ norms as $\gamma \rightarrow \infty$.

SKETCH OF PROOF. In what follows $m=\left(m_{1}, \ldots, m_{N}\right)$ denotes an $N$-tuple of nonnegative integers and similarly $\sigma$ an $N$-tuple of real numbers. We let

$$
\psi_{m}\left(x_{1}, \ldots, x_{N}\right)=\psi_{m_{1}}\left(x_{1}\right) \psi_{m_{2}}\left(x_{2}\right) \cdots \psi_{m_{N}}\left(x_{N}\right) \text { for } x_{1}, \ldots, x_{N} \in G .
$$

As in the two-dimensional case, it is enough to prove the unboundedness of multiplier operators $T$ of the form

$$
T f=\sum_{\sigma \cdot m<0} \hat{f}(m) \psi_{m}
$$

where $\sigma \cdot m=0$ describes a hyperplane unbounded in the first octant and not parallel to any coordinate axes. Such a hyperplane has unbounded intersection with some two-dimensional coordinate plane $P$. Furthermore we may choose $P$ so that this unbounded intersection is a line not parallel to the coordinate axes of $P$. We now restrict our attention to functions $f$ with $\hat{f}(m)=0$ for $m \notin P$. Such functions are constant along coordinate directions of $G^{N}$ not corresponding to coordinate directions of $P$. T acts on such $f$ in the same way as the multiplier of Theorem 1. (Only the case $\beta=0$ is needed.) Thus the unboundedness of $T$ follows from Theorem 2. Our sketch of the proof is finished.

ACKNOWLEDGMENT. The author heartily gives thanks to his advisor Dr. William R. Wade for making this research possible. Dr. Wade and the referee both suggested the proof of Lemma 3 as given.

\section{BIBLIOGRAPHY}

1. C. Fefferman, Inequalities for strongly singular convolution operators, Acta. Math. 124 (1970), 9-36.

2. 
3. __ On the convergence of Fourier series, Bull. Amer. Math. Soc. 77 (1971), 744-745.

4. N. J. Fine, On the Walsh functions, Trans. Amer. Math. Soc. 65 (1949), 372-414.

5. W. Rudin, Fourier analysis on groups, Interscience, New York, 1962.

6. E. M. Stein, On limits of sequences of operators, Ann. of Math. 74 (1961), 140-170.

7. Wo-Sang Young, A note on Walsh-Fourier series, Proc. Amer. Math. Soc. 59 (1976), 305-310.

8. A. Zygmund, Trigonometric series, 2nd ed. (two volumes), Cambridge Univ. Press, Cambridge, 1977.

Department of Mathematics, University of Tennessee, KNoxville, TenNESSEE 37996-1300

Current address: Route 2, Box 207, Buchanan, Tennessee 38222 